Convergences francophones $2.2(2015): 12-24$

http://mrujs.mtroyal.ca/index.php/cf/index

\title{
L'abbé Prévost (1697-1763) et Marc-Antoine Eidous (1724-1790) : esquisse comparée de traducteurs de récits de voyages au XVIIIe siècle
}

\author{
Antoine Eche \\ Mount Royal University/ ICD (Tours)
}

Dans son ouvrage La crise de la conscience européenne, Paul Hazard a relevé le rôle essentiel du voyage dans le basculement psychologique qui fait passer la France de la fin du XVIIe siècle de la stabilité au mouvement. Récits de voyages et romans exotiques connaissent un succès tel que les récits de voyages finissent par occuper le devant de la scène, passant pour être, selon le mot bien connu de Furetière dans son Dictionnaire, " les romans des honnêtes gens ». Pour les auteurs et les lecteurs du XVIIIe siècle, les récits de voyages associent le fameux dyptique horacien du plaisir et de l'instruction. A ce titre, tant les écrits de voyageurs français qu'étrangers intéressent les lecteurs friands de curiosités exotiques, d'informations ethnologiques, historiques, idéologiques ou bien d'informations pratiques destinées à leurs propres pérégrinations (Gannier 726-9). La traduction et la circulation de récits de voyages au XVIIIe siècle, période où « la France traduit sans doute le plus massivement » (Charles 2005: 133), est donc conçue comme une nécessité d'ordre épistémologique.

Dans le champ des études de traduction, on peut remarquer une certaine carence au niveau des études portant sur la traduction des récits de voyages, qui a également été relevée du côté de la critique anglo-saxonne (Martin et Pickford, $2012,3)$. Il sera donc question ici de dresser dans un premier temps un bref état des lieux des études de traduction sur les récits de voyages au XVIIIe siècle.

Cet état des lieux s'articulera sur une autre carence, à savoir la figure de l'abbé Prévost traducteur de récit de voyage. En effet, si la figure du romancier et sa production romanesque bénéficient de tout l'intérêt qu'elles méritent, l'image de Prévost-traducteur dans les études de traduction reste majoritairement confinée à ses traductions des romans anglais de Richardson (Roddier; Jost ; D'hulst: 1990, 107-11 ; Berthiaume ; Charles 2000 ; Moerman 2002 b). Or Prévost n'a pas seulement écrit et traduit des romans mais il a aussi été journaliste, traducteur d'ouvrages historiques et de récits de voyages. Notre propos visera dans cette partie la représentation du traducteur et de son approche traductive au moment de sa carrière où il entreprend la traduction d'une collection anglaise de relations de voyages, la New Collection of Voyages and Travels (1745-1747), éditée par l'Anglais John Green, lui-même traducteur mais également géographe.

Enfin, la figure de Prévost-traducteur de récits de voyages, homme de lettres reconnu par ses pairs, sera comparée à celle de Marc-Antoine Eidous, traducteur polygraphe plus tardif, à la renommée moindre, mais dont le volume de textes traduits relevant de la littérature géographique peut largement être comparé à celui de Prévost. A l'heure où certains traductologues incitent à s'intéresser en premier lieu au traducteur (voir dans ce numéro le texte de Lieven D'hulst), nous espérons donc montrer que la comparaison entre Eidous, traducteur, un temps contributeur à l'Encyclopédie de Diderot et d'Alembert, et l'abbé Prévost, romancier, traducteur 
Convergences francophones $2.2(2015): 12-24$

http://mrujs.mtroyal.ca/index.php/cf/index

et journaliste, s'avère nécessaire afin de mieux cerner l'image ${ }^{1}$ de ces acteurs de la société des Lumières, agents de diffusion d'un savoir jugé indispensable.

\section{Bref état des lieux}

Le manque d'études sur la traduction de récits de voyages peut s'expliquer par l'attrait mais aussi la préférence institutionnalisée longtemps marquées des critiques pour la fiction. Or depuis les années 1980, la critique du récit de voyage a fini par imposer ce dernier en tant que genre, en parallèle à l'anoblissement des genres factuels en général et de l'autobiographie et de l'histoire en particulier. La cause principale de ce changement de perception serait à chercher du côté des effets de la décolonisation et de la critique du colonialisme (Holtz 2-7), du développement du structuralisme en anthropologie sous l'impulsion de Claude Lévi-Strauss mais aussi de l'apparition du 'spatial turn' à la fin des années 1980 dans le domaine de la géographie culturelle sous la plume notamment de Denis Cosgrove. Echappant donc à une approche critique pour qui le récit de voyage n'était qu'un véhicule documentaire, ce genre est maintenant étudié par les littéraires et historiens des mentalités (Roche) dans une optique volontiers pluridisciplinaire, rendant par là même justice à la nature même de ce genre hybride que distingue sa haute capacité à intégrer différents discours (Pasquali). Bref, le récit de voyage relève donc de ce que l'on nomme la littérature, fut-elle entendue dans sa plus large acception.

Cette reconnaissance tardive explique ainsi pourquoi les spécialistes de la traduction au XVIIIe siècle, et sous l'Ancien régime en général, ne se sont guère penchés sur ce sujet, préférant débattre des genres consacrés à cette période (poésie, théâtre) et d'autres en plein développement mais reconnus plus tardivement (le roman). Ainsi, si l'on a pu repérer depuis les années 1970 un « essor des études sur la traduction » où la part allouée au XVIIIe siècle "y occupe une place de choix » (Charles 2005: 133), force est de constater la carence énoncée plus haut, en particulier pour la période qui nous occupe ici. Certes, certains travaux de synthèse ont récemment vu le jour, témoignant ainsi de la jonction entre d'un côté l'institutionnalisation du genre et de l'autre le renouvellement d'une discipline (Cointre, Martin et Pickford 2012 et 2013, Gannier), mais aussi témoignant de la réaction suscitée par la prépondérance des études sur le genre romanesque avec l'émergence d'études portant sur les genres non romanesques (Charles, 135). Toutefois ces travaux ont paru de manière isolée, sans que leurs auteurs ne signalent l'existence de travaux contemporains ou antérieurs, œuvrant dans une sorte d'isolement intellectuel qui n'est pas sans rappeler l'isolement physique du saint patron des traducteurs.

On peut diviser les travaux sur la production de traductions de récits de voyages sous l'Ancien régime en deux tendances plus ou moins

\footnotetext{
${ }^{1}$ Notre démarche peut rappeler celle qu'Agnes Whitfield expose pour élaborer le portrait du traducteur ou de la traductrice $(2012,176)$. Les limites de notre exercice étant ce qu'elles sont, nous avons restreint notre approche à quelques éléments biographiques pertinents et à un corpus stabilisé dans lequel le traducteur se donne à voir (volontairement ou non) dans son approche de la traduction au sein d'une culture donnée, ici celle de la France des Lumières.
} 
contemporaines: ${ }^{2}$ une première tendance anglo-saxonne se manifeste par la parution en 2012 du volume collectif Travel Narratives in Translation, 1750-1830. Nationalism, Ideology, Gender, édité par Alison E. Martin et Susan Pickford, entreprise prolongée avec la parution en 2013 d'un numéro spécial de la revue en ligne inTRAlinea intitulé Travel Writing and Translation. ${ }^{3}$ Une seconde tendance, française, a vu le jour en 2014 avec la parution du premier volume de l'Histoire des traductions en langue française. Portant sur la période XVIIe-XVIIIe siècle, cette histoire contient un chapitre rédigé par une spécialiste de la littérature des voyages sur la traduction des récits de voyages. ${ }^{4}$

Dans les deux cas, il est intéressant d'y remarquer la faible présence, voire parfois l'absence totale, de références critiques à des travaux spécialisés portant sur la traduction des récits de voyages, preuve de la relative nouveauté de ce champ d'investigation et aussi, peut-être, de l'isolement souhaité ou non de certains. Là s'arrêtent les similitudes car tandis que les deux chapitres de dictionnaires proposent un panorama descriptif des raisons motivant ces traductions, des profils de traducteurs, des formes et du style des traductions ainsi que de leur justesse, les deux ouvrages anglais proposent d'interroger les motivations et enjeux idéologiques des traductions de récits de voyages, regroupant ainsi différentes approches du même sujet autour d'une problématique déterminée. Faudrait-il y voir un effet d'une conception distincte de l'approche de l'objet littéraire? Si cette question a déjà fait l'objet d'un débat, ne serait-ce que pour le seul genre du récit de voyage, il ne nous appartient pas de la trancher dans les limites de cet exposé. ${ }^{5}$ Toutefois ces récents travaux apparaissent maintenant incontournables et toute recherche sérieuse sur le sujet se devra d'y faire référence.

\section{Figure de Prévost traducteur de récits de voyages}

\footnotetext{
${ }^{2}$ Nous avons recensé également d'autres travaux collectifs, portant sur d'autres périodes : le deuxième volume de l'Histoire des traductions en langue française (dirigé par Yvres Chevrel, Lieven D'hulst et Christine Lombez) paru en 2012 porte sur le XIXe siècle et contient un chapitre collectif sur les récits de voyages; en 2001 Loredana Polezzi a édité un ouvrage collectif sur la traduction en anglais de récits de voyages italiens contemporains: Translating Travel: Contemporary Italian Travel Writing in English Translation (Aldershot : Ashgate).

${ }^{3} \mathrm{Il}$ faudrait également citer la publication en 2006 de l'ouvrage collectif Travel and Translation in the Early Modern Period sous la direction de Carmine Diase. L'auteure articule une collection d'articles autour des relations entre voyage et traduction dans une acception thématique très large. Elle ne distingue pas entre récits de voyages imaginaires et récits de voyages authentiques. Ceci pose question dès lors que l'on s'intéresse aux modifications éventuelles du contenu documentaire des récits authentiques lors de la traduction.

${ }^{4}$ Nous avons également relevé un article d'Annie Cointre de 2003, « Les Lettres du voyage en Turquie de Lady Mary Wortley Montagu en France ». Cependant l'article n'est pas un article de synthèse mais une étude de cas centrée sur l'épistolaire et sa réception. Signalons également l'article de l'historienne Nicole Pellegrin, «Une traductrice historienne. Louise de Keralio-Robert et les voyageurs anglais ». Toutefois la traduction des deux relations de voyages discutée en détails dans cet article excède les limites chronologiques qui sont les nôtres et relève d'un contexte idéologique bien distinct (l'Empire plutôt que les Lumières).

${ }^{5}$ Voir Charles Forsdick, Feroza Basu et Siobhan Shilton, New Approaches to Twentieth-Century Travel Literature in French: Genre, History, Theory. Francfort-sur-le-Main : Peter Lang, 2006. 1415 ; Jan Borm, "Defining Travel: On the Travel Book, Travel Writing and Terminology ». Ed. Glenn Hooper and Tim Youngs. Perspectives on Travel Writing. Aldershot: Ashgate, 2004. 13-26.
} 
Convergences francophones $2.2(2015): 12-24$

http://mrujs.mtroyal.ca/index.php/cf/index

Par la place particulière qu'occupe la littérature de voyages dans l'épistémè du siècle des Lumières, force est de reconnaître que tout traducteur incarne par la force des choses la figure d'un traducteur-passeur de savoirs. Mais cette définition paraît tout de suite bien insuffisante lorsqu'on considère que l'acte de traduction, comme le regard du voyageur, n'est jamais neutre. Les divers travaux mentionnés précédemment montrent tous qu'il en est de la traduction du récit de voyage comme celle de la fiction et que chaque traduction contient son lot d'omissions, d'ajouts et/ou de commentaires (Gannier 744-52). Certaines pratiques sont imposées par la nature même du type de publications à traduire. En effet, la compilation de plusieurs récits se distingue de la relation unique déjà par la réduction quasi systématique des textes originaux. Et le passage à la langue cible se fait au crible d'une nationalisation du texte qui vient se superposer à des modifications antérieures. Le traducteur de récits de voyages est donc plutôt manipulateur, voire créateur comme dans le cas de l'abbé Prévost.

Si l'auteur de Manon Lescaut fait encore partie de la mémoire du plus grand nombre, peu en revanche savent que l'abbé Prévost fut également traducteur de romans anglais et encore moins savent qu'il est l'auteur de la traduction de plusieurs textes brefs et divers publiés dans son journal, le Pour et Contre, publié de 1733 à 1740, ainsi que de deux ouvrages historiques, à savoir le premier tome de l'Historia sui temporis de M. de Thou en 1731 et l'Histoire de Cicéron de l'Anglais Middleton en 1743. La traduction de l'Histoire générale des voyages (désormais $H G V$ ) reste quantitativement l'œuvre traduite par Prévost la plus conséquente: elle est composée de quinze volumes in-quarto (chaque volume contient environ 700 pages) publiée de 1746 à 1759. L'histoire de l'édition de l' $H G V$ se distingue par trois phases éditoriales et c'est seulement la première qui nous importe ici avec la traduction des quatre volumes de la collection anglaise correspondant aux sept premiers tomes de l'édition Didot ainsi qu'une soixantaine de pages du huitième tome (1746-1750). ${ }^{6}$ Compte tenu des limites de cet article, nous ne nous intéresserons qu'à un seul aspect de la figure, au sens propre comme au figuré, de Prévost-traducteur.

Nous avons dénombré trois études faisant référence au travail de traduction effectué par Prévost sur cette collection. Un premier article ambitionne de « dresser une typologie générale » à partir de l'exemple du traitement des voyages portugais dans l'HGV (Albertan-Coppola 1999). La seconde étude est celle d'Ellen Moerman, qui a déjà travaillé sur les multiples traductions faites par Prévost dans le Pour et Contre, et qui propose elle-aussi un travail ciblé mais sans ambition généraliste sur les traductions de récits de pirates dans cette collection (2002). La troisième et la plus récente s'intéresse aux effets de la traduction sur la représentation de l'Inde et des Indiens (D'Souza). Dans l'ensemble, ces travaux montrent sur des échantillons précis les modifications apportées par Prévost au texte source mais aussi ce qui n'a pas été modifié ainsi que la réaction d'une partie

${ }^{6}$ Viennent ensuite une période de transition dans les tomes huit à onze (1750-1753) et enfin la période des tomes douze à quinze où Prévost concrétise le plan qu'il avait en tête depuis le début du projet (1754-1759). Voir Duchet (147); Albertan-Coppola (1995). Sur l'ensemble du projet, voir la très bonne synthèse de Jomand-Baudry. 
Convergences francophones $2.2(2015): 12-24$

http://mrujs.mtroyal.ca/index.php/cf/index

du lectorat (D'Souza). Toutefois l'activité traductive antérieure de Prévost n'y est pas abordée, ce qui tend quelque peu à biaiser l'image partielle du traducteur qui peut se dégager de ces travaux. ${ }^{7}$

Dans cette activité, Prévost bénéficie de sa réputation de romancier et de styliste apprécié, tant chez les lecteurs et lectrices telle Mme du Deffand que dans les journaux (Sgard 228-31). Il est à noter cependant que les traductions antérieures d'ouvrages complets comme par exemple l'Histoire de Cicéron, que nous distinguons de celles faites pour le Pour et Contre, ${ }^{8}$ ne mentionnent pas le nom du traducteur. Cette mention figure généralement dans les avis de publication diffusé dans la presse, sans que cela ne constitue une obligation. La question de la visibilité $\mathrm{du}$ traducteur se pose donc ici à la jonction d'un épitexte capricieux et de sa réception au sein des « institutions de la vie littéraire » (Viala).

Toutefois le cas de l' $H G V$ attire l'attention. En effet, et en dépit de toute mention au nom du traducteur, la figure (au sens propre) de Prévost y apparaît bel et bien dans un portrait placé en regard de la page de titre. La position de son visage, tourné en direction de la page de titre, et la visée plongeante de son regard dénotent la position de supériorité du sujet regardant face à l'ouvrage. Visuellement, Prévost affiche donc une autorité éditoriale et auctoriale (Eche). Le commentaire renvoyant à cette image confirme cette idée. Feignant vraisemblablement l'ignorance, Prévost blâme son libraire mais n'en cache pas moins son plaisir:

Je ne sais par quelle fantaisie il a plu au libraire de me faire paraitre à la tête de mon livre. Mes amis sont témoins de ma résistance. Si la faiblesse que j'ai eue de me rendre est une faute, je la crois fort bien réparée par l'excellence du portrait, qui est l'ouvrage du célèbre Smith ( $H G V$ I: xvii). ${ }^{9}$

En présentant l' $H G V$ comme "son" livre, Prévost non seulement affirme d'emblée sa paternité textuelle mais aussi sa conception de la traduction et de l'édition. Grâce à son traducteur, le texte traduit devient œuvre nouvelle. En replaçant cette traduction dans le cheminement traductif de Prévost, on remarque que ce dernier avait déjà exprimé par cette formule possessive cette même substitution d'autorité dans sa traduction de la vie de Cicéron de l'Anglais Middleton. Dans une longue préface, Prévost vantait les mérites de l'auteur anglais mais aussi ses faiblesses pour mieux souligner le sérieux de la démarche traductrice qu'il avait héritée de son séjour chez les bénédictins de Saint-Maure ainsi que sa propre conception de la traduction. Son rôle de traducteur n'impliquait pas juste la simple traduction littérale du texte anglais mais également une réécriture corrective et explicative nécessaire pour ce qu'il nommait « mon histoire ». ${ }^{10}$

\footnotetext{
${ }^{7}$ Dont le but, rappelons-le, n'est pas de mettre en avant la figure du traducteur mais des pratiques et des effets textuels.

${ }^{8}$ Sur ces dernières voir Charles (1992, 203-235) et Moerman (1998).

${ }^{9}$ Le premier chiffre renvoie au tome, le second à la page. Sur les portraits de Prévost, voir Sgard (2006, 215-217).

${ }^{10}$ Prévost, après avoir salué les qualités d'historien de Middleton, souligne certains manques, notamment en ce qui concerne des informations permettant de mieux saisir le contexte de la période à laquelle vivait Cicéron. Il juge que les ajouts factuels ne sont pas inutiles "pour l'intelligence d'une infinité d'endroits de notre histoire », Histoire de Cicéron, tirée de ses écrits et des
} 
Convergences francophones $2.2(2015): 12-24$

http://mrujs.mtroyal.ca/index.php/cf/index

La paternité de l' $H G V$ semble cependant difficile à porter ou du moins, à faire accepter. Jouant du topos de la modestie affectée, Prévost fait montre dans le premier «Avertissement du traducteur» d'une attitude quelque peu paradoxale. Après avoir indiqué qu'il avait ajouté quelques relations au corpus déjà existant, ${ }^{11}$ il en vient à se plaindre de son obligation à suivre le plan des Anglais :

... je ne suis pas libre de cacher mes regrets sur la nécessité où je me trouve de suivre pas à pas des compilateurs étrangers, et de m'assujettir servilement à leur plan. Non que je le condamne : mais n'en connaissant que ce qu'ils en ont annoncé dans leur préface et dans leur introduction, je suis forcé d'attendre la publication de chaque volume pour juger de l'exécution, sans pouvoir jamais espérer de faire jamais à l'ordre général aucun changement qui convienne aux idées de ma patrie. Aussi n'ai-je pas d'autre gloire à prétendre ici que celle d'une simple traduction. ( $H G V$ I: xiv)

Le traducteur ne peut donc faire reconnaitre ses talents, qui ne se manifestent pas dans la traduction du texte mais dans l'organisation même de l'ouvrage. Ce faisant, Prévost renvoie directement au processus originel de la composition de cette collection qui relève en plein de la sphère auctoriale. Cette figure topique va toutefois être mise à mal dans les discours préfaciels des volumes qui suivront. On y constate que Prévost oscille entre l'affirmation de la supériorité de son approche vis-à-vis de celle de son homologue anglais qui se manifeste dans le texte par l'ajout de références et de commentaires en notes de bas de pages, mais aussi une attitude défensive qui surgit dès lors que paraît en Hollande une édition concurrente à la sienne. Cette édition, reposant sur la traduction de Prévost, se veut néanmoins corrective, et son auteur reproche au Français d'avoir tronqué l'original par endroits ainsi que d'avoir commis des erreurs, et donc d'avoir altéré la dimension factuelle des relations. ${ }^{12} \mathrm{~L}^{\prime} H G V$ sera néanmoins bien reçue, comme en témoigne son utilisation répétée par Voltaire, Rousseau, Diderot ou Buffon et les commentaires des journalistes de l'époque, et l'abandon du projet initial par son auteur en 1747 libérera Prévost de la contrainte du texte source, pour, bien sûr, en apporter d'autres.

\section{Figure de Marc-Antoine Eidous}

S'il n'a pas paru utile de rappeler précisément qui était Antoine-François Prévost, il l'est peut-être plus pour Marc-Antoine Eidous. A la différence de l'abbé Prévost, Eidous ne débute pas sa carrière dans les lettres en étant romancier et n'obtiendra jamais une notoriété comparable. Figure de l'ombre de la production culturelle des Lumières, Eidous est d'abord ingénieur dans l'armée espagnole avant

monuments de son siècle; avec les preuves et des éclaircissements. Paris : Didot, 1743. xiv. (je souligne).

11 « Notre premier livre est enrichi de quelques autres relations, dont l'autorité ne demande pas moins d'être établie », $H G V$, I, Avertissement du traducteur, xiii.

${ }^{12}$ Histoire générale des voyages [...] Nouvelle édition, revue sur l'original anglois [...]. La Haye : Pierre de Hondt, 1747, I : xvii-xx. Sur ce texte et la réaction qu'il a suscitée chez Prévost, voir notre article déjà cité. 
de devenir traducteur à Paris. On lui reconnaît une quarantaine de traductions aux genres divers: philosophie, médecine, roman et littérature géographique. Son amitié avec Diderot lui vaudra de collaborer notamment à l'Encyclopédie pour les articles touchant à l'équitation, la maréchalerie et à l'héraldique (Kafker, 128-130). Polyglotte, Eidous a traduit depuis cinq langues différentes vers le français, travaillait vite et semblait être apprécié des éditeurs (Donato, 30). Toutefois, les journalistes comme Grimm dans la Correspondance littéraire par exemple, ainsi que Diderot, étaient beaucoup moins tendres. Deux études lui ont été consacrées : la première en 1980 pour sa traduction d'un ouvrage de Daniel Defoe, A General History of Discoveries and Improvements (1726-1727) traduit sous le titre Histoire des principales découvertes faites dans les arts et les sciences (1767) (Wilkie); la seconde, plus récente, porte directement sur le rôle de Eidous en tant que traducteur de récits de voyages (Donato).

Pour des raisons de cohérence générique, nous avons décidé de cibler notre comparaison sur les écrits géographiques traduits par Eidous, en incluant récits de voyages et traités historico-géographiques. L'élargissement de notre corpus à la littérature géographique ne nous semble pas incongru dans la mesure où l'Histoire des voyages de Prévost rassemble déjà récits de voyages et autres textes géographiques et historiques, tous soumis aux prescriptions génériques du récit factuel canonique de la période : le récit historique. ${ }^{13}$

Les titres des ouvrages relevant de la littérature géographique montrent d'emblée les traces de la visibilité du traducteur au niveau paratextuel, à l'inverse du titre de l' $H G V$ de Prévost. ${ }^{14}$

- Histoire naturelle, civile et géographique de l'Orénoque et des principales rivières qui s'y jettent [...] par le Père Joseph Gumilla [...] Traduite de l'espagnol sur la seconde édition par M. Eidous ; cidevant ingénieur des armées de S.M.C., 3 vols, Avignon : Jean Messy, 1758.

- Voyages depuis St. Pétersbourg en Russie dans diverses contrées de l'Asie [...] Par Jean Bell d'Antermony. Traduits de l'Anglois par M ***., Paris : Robin, 1766.

- Histoire des colonies européennes dans l'Amérique [...]. Traduite de l'Anglois de M. William Burck (sic) par M.E., 2 vols, Paris : Merlin, 1767.

- Histoire naturelle et civile de la Californie, contenant une description exacte de ce pays [..., ] Traduite de l'Anglois par M. E. **., 3 vols, Paris : Durand, 1767.

- Histoire de Kamtschatka, des îles Kurilski et des contrées voisines [...] Traduite par M. E***, 2 vols, Lyon : Benoît Duplain, 1767.

\footnotetext{
${ }^{13}$ Donato ouvre son étude à certains textes de fictions relevant plus ou du moins du thème du voyage (35-37). Nous préférons (pour l'instant) conserver l'homogénéité d'un corpus de textes factuels.

${ }^{14}$ Cette liste a été établie d'après l'article de Donato et les 68 recensions de traductions tirées du catalogue de la $\mathrm{BnF}$ (data.BnF.fr) en utilisant l'entrée "Marc-Antoine Eidous » [consulté le 15/09/2015]. Elle contient trois titres de plus que celle établie par Donato, à savoir: l'Histoire de Kamtschatka, l'Histoire de la Russie et l'Histoire de l'Amérique.
} 
Convergences francophones $2.2(2015): 12-24$

http://mrujs.mtroyal.ca/index.php/cf/index

- Histoire de la Nouvelle York [...] Par William Smith. Traduite de l'Anglois par M. E***., Londres : 1767.

- Histoire de la Russie [...] par Michel Lomonossow [...]. Traduit de l'Allemand par M.E***., Paris : Guillyn, 1769.

- Voyages dans le Levant [...] par Frédéric Hasselquist [...] Traduits de l'Allemand par M.***., Paris : Saugrain le jeune, 1769.

- Histoire de l'Amérique, par Guillaume Robertson [...] Traduite par M. E..., 4 vols, Maastricht: Jean-Edme Dufour et Philippe Roux, 1777.

Les initiales du traducteur apparaissent en effet dans les titres, sous trois formes principales: la mention «M. Eidous » apparaît dans un titre; la mention «M.E. » et ses variantes «M. E. **» et «M.E. ***» dans six titres ; celle, plus cryptique, « M. ***» dans deux titres. Pour le lecteur informé de la période par les annonces de publication diffusées dans la presse ou par le bouche-à-oreille, il est aisé de reconnaître le traducteur derrière ces initiales. Ainsi, la traduction du récit des Voyages depuis St Petersbourg est attribuée à Eidous dans la Correspondance littéraire du 15 septembre 1766 (153). D'autres éléments péritextuels peuvent aussi contribuer à l'identification du traducteur. Ainsi sa traduction de l'Histoire de Kamtschatka de Stepan Krasheninnikov d'après la traduction anglaise donnée par James Grieve (The History of Kamtschatka, and the Kurilski Islands, Gloucester: Raikes, 1764) est plus complète car Eidous est détenteur du privilège d'impression accompagnant la publication. Cette mention est redoublée dans la mention de la cession de ce privilège à l'éditeur Deplain à Lyon (n.p.).

On remarque également une autre différence significative relevant de la visibilité de la figure, au sens propre, de Eidous : moins connu que Prévost, MarcAntoine Eidous n'aura pas de portrait figurant en regard des très rares textes liminaires qu'il fournira à ses lecteurs.

A cet égard, deux textes attirent plus particulièrement notre attention. Sa traduction de l'Histoire naturelle, civile et géographique de l'Orénoque se distingue déjà par la mention sur la page de titre de son titre d'ingénieur des armées, illustration maximale de son autorité en la matière. Comme Prévost, Eidous semble revendiquer la paternité du texte traduit dans l'avertissement du traducteur qu'il y joint :

La préface qu'on vient de lire suffit pour mettre le lecteur au fait de mon ouvrage; ainsi je m'en dispenserai d'en donner une seconde, qui m'obligerait à des redites inutiles, et je me contenterai d'avertir les personnes entre les mains desquelles cette histoire pourra tomber, que dans le dessein de la rendre aussi exacte qu'utile, j'ai voulu éclaircir un doute, dont la solution importe beaucoup aux progrès de la géographie et à la connaissance du globe que nous habitons (n.p. c'est nous qui soulignons)

Si la polysémie du mot «ouvrage» peut s'avérer problématique, précisons que dans ce texte, Eidous utilise deux fois en tout le mot ouvrage, la seconde fois pour désigner explicitement le texte original, ce qui permet de penser qu'il en fait de même dès la première fois. De plus, Eidous se dégage des responsabilités d'écrire 
Convergences francophones $2.2(2015): 12-24$

http://mrujs.mtroyal.ca/index.php/cf/index

une préface, prérogative qui est traditionnellement celle de l'auteur, ce qui indique clairement que le cas échéant, il serait parfaitement à même de remplir ce rôle. ${ }^{15}$

Marc-Antoine Eidous n'accompagne pas systématiquement ses traductions d'avertissements qui permettraient de mieux apprécier la perception qu'il a de sa fonction. Il en est ainsi pour les autres titres de notre liste, ${ }^{16}$ à l'exception notable de l'Histoire de la Russie, traduite en français à partir d'une version allemande due au baron d'Holbach. L'ouvrage contient ainsi les deux avis des deux traducteurs. Il ne faut pas chercher cette fois une quelconque revendication de paternité dans l'avis rédigé par Eidous. Après avoir déploré le manque de sérieux des ouvrages historiques actuels et notamment des abrégés ainsi que la piètre qualité des informations disponibles sur la Russie (v-ix), il souligne en fait la qualité de l'auteur traduit et du contenu de son histoire (ix-X) ainsi que la perspicacité du traducteur du russe vers l'allemand (x-xi). Eidous se montre en fait plutôt sous les traits de la figure plus attendue du traducteur-passeur, intermédiaire utile à l'édification du savoir des Lumières : à ce titre, il joint au texte «... deux cartes de la Russie, en faveur de ceux qui ne connaissent point le pays » (x-xi). Dans le même temps, il se montre soucieux de conserver une certaine altérité textuelle en choisissant de ne pas franciser les noms propres des villes et des peuples mentionnés, ce afin de ne pas « les défigurer en leur donnant une terminaison française » (xi). Notons que la question de la toponymie n'est pas propre à Eidous. Dans son avertissement du traducteur, Prévost avait lui aussi relevé cette difficulté, en faisant toutefois valoir une attention corrective concernant la variété des orthographes des toponymes étrangers. Il ne s'agissait pas tant de préserver l'altérité de la notion que de garantir une cohérence graphique garante de la valeur informative du texte ( $H G V, \mathrm{I}, \mathrm{xv}-$ xvi).

Si onze années séparent la publication de ces deux traductions par Eidous, rien ne laisse cependant supposer que le changement d'attitude noté dans le second liminaire mentionné supplantera les revendications auctoriales remarquées dans le premier. Il faudrait étendre cette recherche au reste de ses traductions afin de dénombrer plus précisément les figures à l'œuvre dans sa carrière de traducteur. Toutefois, et dans les limites du corpus examiné, il convient de signaler qu'à la différence de Prévost, Eidous ne manifeste aucune gêne vis-à-vis de la structure des récits évoqués ni ne critique les auteurs. Il ne dit pas non plus vouloir accommoder les récits au goût français. Cette attitude s'explique peut-être par la différence structurelle et de visée entre une « simple » relation et une compilation de relations

\footnotetext{
${ }^{15} \mathrm{Si}$ Donato relève aussi cette construction d'une figure auctoriale dans ce texte, ses arguments visent plutôt à mettre en valeur la dimension corrective de la démarche d'Eidous.

${ }^{16}$ A ce propos, nous avons relevé une erreur dans l'article de Donato qui affirme que la préface accompagnant la traduction de l'Histoire naturelle et civile de la Californie est de la main d'Eidous (Donato, 34). Or cette préface figure déjà dans le texte anglais dont s'est servi Eidous mais pas dans l'original espagnol, publié d'ailleurs en 1757 et non en 1739 comme indiqué par Donato. Eidous traduit en fait la préface du traducteur anglais ainsi que l'ensemble du texte principal et des annexes fournies dans le dernier volume, textes également présents dans la version espagnole. Voir A Natural and Civil History of California ... Translated from the original Spanish of Miguel Venegas, A Mexican Jesuit, published at Madrid, 1758. 2 vols. Londres: James Rivington et James Fletcher, 1759 ; Noticia de la California y de su conquista temporal y espiritual .... 3 vols. Madrid : Imprenta de la viuda de Manuel Fernandez, 1757.
} 
mais aussi peut-être par un parcours intellectuel et une ambition littéraire (au sens large) distincts.

L'esquisse comparée des figures de ces deux traducteurs à la renommée distincte laissent bien paraître certaines similarités, notamment, et au-delà de leur intérêt pour le genre de la littérature géographique (pourrait-il en être autrement au XVIIIe siècle ?), une tendance à la spécialisation. Cette brève étude met également au jour les revendications auctoriales de ces traducteurs. Toutefois, on peut observer que cette attitude va disparaître rapidement chez Eidous pour des raisons encore méconnues : faute de temps pour écrire une préface ? préface non souhaitée par l'éditeur? volonté de devenir invisible ? Chez Prévost, notons également le rôle contraignant de la concurrence qui va le forcer à plusieurs reprises à justifier son approche de la traduction ainsi que son aveu d'un soulagement ressenti à l'annonce par Green de l'arrêt du projet en Angleterre ( $H G V$, VIII, n.p), le dégageant donc de son rôle de traducteur.

Souhaitons pour finir que nous ayons pu montrer l'intérêt d'une approche comparée des figures des traducteurs des récits de voyages au XVIIIe siècle à un moment où l'approche historique de la traduction francophone connaît un essor certain. Alors que le champ des études sur la littérature des voyages s'agrandit grâce à la considération maintenant accordée au rôle de la traduction et des traducteurs tant dans la fiction que dans les genres factuels, il s'avère également nécessaire d'interroger la recherche produite sur ce sujet dans d'autres langues. Il ne s'agirait pas que le critique devienne le doublon de la figure caricaturale du traducteur. 
Convergences francophones $2.2(2015): 12-24$

http://mrujs.mtroyal.ca/index.php/cf/index

\section{Bibliographie}

Albertan-Coppola, Sylviane. «Les voyages portugais dans l'Histoire générale des voyages de l'abbé Prévost. » Dix-huitième siècle 31 (1999): 491-506.

---. « Constitution, métamorphose et célébration du savoir.» La Revue française, Numéro spécial électronique : La Culture des voyageurs à l'âge classique. $<$ http://revuefrancaise.free.fr/Albertan.htm.> Consulté le 18 septembre 2015.

Berthiaume, Pierre. «Prévost traducteur de Frances Sheridan.» La Traduction des langues modernes au XVIIIe siècle ou «La Dernière Chemise de l'Amour». Ed. Annie Rivara. Paris: Honoré Champion, 2002. 57-84.

Charles, Shelly. Récit et réflexion: poétique de l'hétérogène dans Le Pour et contre de Prévost. Studies on Voltaire and the 18th-Century 298. Oxford: Voltaire Foundation, 1992.

---. « Traduire au XVIIIe siècle. » SVEC 10 (2005): 133-147.

---. « De la traduction au pastiche: 1'Histoire du chevalier Grandisson. » Eighteenth Century Fiction 13.1 (2000): 19-40.

Chevrel, Yves, Annie Cointre et Yan-Maï Tran-Gervat. dir. Histoire des traductions en langue française, XVIIe-XVIIIe siècles. Paris: Verdier, 2014.

Cointre, Annie. « Les Lettres du voyage en Turquie de Lady Mary Wortley Montagu en France. » La Traduction des genres non romanesques au XVIIIe siècle. Ed. Cointre, Annie et Annie Rivara. Metz: Centre d'études de la traduction, 2003. 233-48.

Cointre, Annie et Annie Rivara. La Traduction des genres non romanesques au XVIIIe siècle. Metz: Centre d'études de la traduction, 2003.

D'hulst, Lieven. Cent ans de théorie française de la traduction: de Batteux à Littré, 1748-1847. Lille: Presses Universitaires du Septentrion, 1990.

Donato, Clorinda. " The Travels and Translations of Marce-Antoine Eidous. " Travel Narratives in translation, 1750-1830. Nationalism, Ideology,

Gender. Dir. Martin, Alison et Pickford, Susan. Londres: Routledge, 2012. 28-41.

D’Souza, Florence. « Les représentations de l'Inde et des Indiens dans l'Histoire générale des voyages de l'Abbé Prévost : le rôle de la traduction en français à partir d'un original en anglais dans l'accumulation des saviors. " Annales historiques de la Révolution française 375 (2014) : 27-48.

Duchet, Michèle. " L'Histoire des voyages: originalité et influence. » L'abbé Prévost. Actes du colloque d'Aix-en-Provence, 20-21 décembre 1963. Aixen-Provence: Publication des Annales de la Faculté des Lettres, 1965. 14754.

Eche, Antoine. «Contraintes et libertés de l'autorité éditoriale dans l'Histoire générale des voyages de l'abbé Prévost (1746-1759). » L'autorité dans le monde des lettres. dir. Élisabeth Gavoille, Marie-Paule de Weerdt-Pilorge et Philippe Chardin. Paris: Kimé, 2015.

Gannier, Odile. « Récits de voyages. » Histoire des traductions en langue française, XVIIe-XVIIIe siècles. dir. Yves Chevrel, Annie Cointre et YanMaï et Tran-Gervat. Paris: Verdier, 2014. 723-68.

Green, John. Collection of Voyages and Travels. 4 vols. Londres: Astley, 1745- 
Convergences francophones $2.2(2015): 12-24$

http://mrujs.mtroyal.ca/index.php/cf/index

47.

Grimm Friedrich Melchior et Denis Diderot. Correspondance littéraire, philosophique et critique de Grimm et Diderot depuis 1753 jusqu'en 1790. Vol. V (1766-1768). Paris: Furne, 1829.

Hazard, Paul. La crise de la conscience européenne 1680-1715. Paris: Le livre de poche, 1994. [1935]

Holtz, Grégoire et Masse, Vincent. «Étudier les récits de voyage: bilan, questionnements, enjeux. » Arborescences : revue d'études françaises 2 (2012) : 1-30.

Jomand-Baudry, Régine. «L'aventure éditoriale de l'Histoire des voyages de J.A Prévost d'Exiles. » Cahiers Prévost d'Exiles 10 (1994): 3-12.

Jost, François. «L'Abbé Prévost traducteur de Richardson. » Revue des langues vivantes XXXIX.4 (1973) : 346-59.

Kafker, Frank et Serena Kafker. The Encyclopedists A Individuals: A

Biographical Dictionary of the Authors of the Encyclopédie. SVEC 257, 1988.

Martin, Alison et Susan Pickford. Travel Narratives in Translation, 1750-1830. Nationalism, ideology, Gender. Londres: Routledge, 2012.

---. inTRAlinea Special Issue: Translating 18th and 19th Century European Travel Writing (2013).

$<$ http://www.intralinea.org/specials/travelwritetrans $>$. [consulté le 15 septembre 2015]

Moerman, Ellen. L'abbé Prévost traducteur, ou la tyrannie du bon goût : l'époque $d u$ « Pour et Contre» (1733-1740). Thèse, Université Montpellier III, Paul Valéry, 1998.

---. « Les pirates dans l'Histoire générale des voyages : traduction commerciale, romanesque et philosophique. " Les tyrans de la mer: pirates, corsaires et flibustiers. dir. Sylvie Requemora et Sophie Linon-Chipon. Paris: Presses de l'université Paris-Sorbonne, 2002a. 231-242.

---. « Traduire le geste: Sidney Bidulph, Sheridan, Prévost et Robinet ». La Traduction des langues modernes au XVIIIe siècle ou "La Dernière Chemise de l'Amour». dir. Annie Rivara. Paris: Honoré Champion, $2002 \mathrm{~b}$. 85-108.

Pasquali, Adrien. Le Tour des horizons: Critique et récits de voyage. Paris: Klincksieck, 1994.

Pellegrin, Nicole. « Une traductrice historienne. Louise de Keralio-Robert et les voyageurs anglais. » Femmes écrivains à la croisée des langues 1700-2000. dir. Agnese Fidecaro, Henriette Partzsch, Susan van Dijk et Valérie Cossy. Genève: Metis Presses, 2009. 67-90.

Prévost, Antoine-François. Histoire générale des voyages. 15 vols. Paris: Didot, 1746-1759.

Roche, Daniel. Humeurs vagabondes. De la circulation des hommes et de l'utilité des voyages. Paris: Fayard, 2003.

Roddier, Henri. « L'Abbé Prévost et le problème de la traduction au XVIIIe Siècle. » 1'Association internationale des études françaises 8 (1958):17381 . 
Convergences francophones 2.2 (2015) : 12-24

http://mrujs.mtroyal.ca/index.php/cf/index

Sgard, Jean. Vie de Prévost 1697-1763. Québec: Presses de l'Université Laval, 2006.

Viala, Alain. « Effets de champ et effets de prisme. » Littérature 70 (1988): 6471.

Whitfield, Agnes. « Méthode et pratique du portrait: sur les traces des traducteurs. » Romanica Wratislaviensia 59 (2012): 175-84.

Wilkie, Everett. « Eidous' Translation of Defoe's A General History of Discoveries and Improvements ». Papers of the Bibliographical Society of America 74 (1980): 67-70. 